Original Research Paper

\title{
Coupled Pettis-Hadamard Fractional Differential Systems with Retarded and Advanced Arguments
}

\author{
${ }^{1}$ Saïd Abbas, ${ }^{2}$ Mouffak Benchohra and ${ }^{3}$ Seenith Sivasundaram \\ ${ }^{1}$ Laboratory of Mathematics, Geometry, Analysis, Control and Applications, \\ Tahar Moulay University of Saïda, P.O. Box 138, EN-Nasr, 20000 Saïda, Algeria \\ ${ }^{2}$ Laboratory of Mathematics, Djillali Liabes University of Sidi Bel-Abbes, P.O. Box 89, Sidi Bel-Abb es 22000, Algeria \\ ${ }^{3}$ Department of Mathematics, College of Engineering Science and Mathematics, Daytona Beach, FL 32114, USA
}

Article history

Received: 25-12-2017

Revised: $10-01-2018$

Accepted: 03-02-2018

Corresponding author: Seenith Sivasundaram

Department of Mathematics,

College of Engineering Science

and Mathematics, Daytona

Beach, FL 32114, USA

Email: seenithi@gmail.com

\begin{abstract}
In this study, we present some results concerning the existence of weak solutions for some coupled systems of Hadamard fractional differential equations involving the both retarded and advanced arguments. Our results are obtained by using fixed point theory and the technique of measure of weak noncompactness.
\end{abstract}

Keywords: Functional Differential Equation, Left-Sided Mixed Pettis Hadamard Integral of Fractional Order, Pettis Hadamard Fractional Derivative, Coupled System, Retarded, Advanced, Weak Solution, Fixed Point

\section{Introduction}

Fractional differential equations have recently been applied in various areas of engineering, mathematics, physics and bio-engineering and other applied sciences (Hilfer, 2000; Tarasov, 2010). For some fundamental results in the theory of fractional calculus and fractional differential equations we refer the reader to the monographs (Abbas et al., 2012; 2015; Ahmad and Ntouyas, 2015; Kilbas et al., 2006; Thiramanus et al., 2014; Zhou, 2014). There has been a significant development in fractional differential and integral equations with delay in recent years; (Abbas et al., 2012; 2015; Ahmad et al., 2017; Samko et al., 1993) and the references therein.

The measure of weak noncompactness is introduced by De Blasi (1977). The strong measure of noncompactness was considered in many papers; (Banas and Goebel, 1980; Akhmerov et al., 1992; Alvarez, 1985; Benchohra et al., 2008; Guo et al., 1996; Reich, 1973; 1972) and the references therein. Benchohra et al. (2008; O'Regan, 1999) the authors considered some existence results by applying the techniques of the measure of noncompactness. Recently, several researchers obtained other results by application of the technique of measure of weak noncompactness; (Abbas et al., 2015; Benchohra et al., 2011;2012) and the references therein.

In (Agarwal et al., 2016), the authors studied the existence and uniqueness of solutions for boundary value problems of Hadamard-type fractional functional differential equations and inclusions involving both retarded and advanced arguments. In (Abbas et al., 2017), the authors discuss the existence of weak solutions for the following boundary value problem for implicit Pettis Hadamard fractional differential equation:

$$
\left\{\begin{array}{l}
u(t)=\phi(t) ; t \in[1-\alpha, 1], \\
\left({ }^{H} D_{1}^{r} u\right)(t)=f\left(t, u_{t},\left({ }^{H} D_{1}^{r} u\right)(t)\right) ; t \in I:=[1, e], \\
u(t)=\psi(t) ; t \in[e, e+\beta] .
\end{array}\right.
$$

Motivated by the previous works, in this study, we discuss the existence of weak solutions for the following coupled system of Pettis-Hadamard fractional differential equations:

$$
\left\{\begin{array}{l}
(u(t), v(t))=\left(\phi_{1}(t), \phi_{2}(t)\right) ; t \in[1-\alpha, 1], \\
\left(\left({ }^{H} D_{1}^{r_{1}} u\right)(t),\left({ }^{H} D_{1}^{r_{2}} v\right)(t)\right) \\
=\left(f_{1}\left(t, u_{t}, v_{t}\right), f_{2}\left(t, u_{t}, v_{t}\right)\right) ; t \in I:=[1, e], \\
(u(t), v(t))=\left(\psi_{1}(t), \psi_{2}(t)\right) ; t \in[e, e+\beta],
\end{array}\right.
$$

where, $\alpha, \beta>0, r_{i} \in(1,2], f_{i}: I \times C[-\alpha, \beta] \times C[-\alpha, \beta] \rightarrow E$; $i=1.2$ are given continuous functions, $\phi_{i} \in C[1-\alpha, 1]$ with $\phi_{i}(1)=0, \psi_{i} \in C[e, e+\beta]$ with $\psi_{i}(e)=0, E$ is a real (or complex) Banach space with norm $\|\cdot\|_{E}$ and dual $E^{*}$, such 
that $E$ is the dual of a weakly compactly generated Banach space $X, C[-\alpha, \beta]$ is the space of continuous functions from $[-\alpha, \beta]$ to $E$ and ${ }^{H} D_{1}^{r_{i}}$ is the PettisHadamard fractional derivative of order $r_{i} ; i=1,2$.

We denote by $u_{t}$ the element of $C[-\alpha, \beta]$ defined by:

$$
u_{t}(s)=u(t+s) ; t \in[-\alpha, \beta] .
$$

This paper initiates the study of coupled systems of Hadamard fractional differential equations under weak topologies.

\section{Preliminaries}

Let $C(I)$ be the Banach space of all continuous functions $w$ from $I$ into $E$ with the supremum (uniform) norm:

$$
\|w\|_{\infty}:=_{t \in I}\|w(t)\|_{E}
$$

As usual, we denote by $A C(I)$ the space of absolutely continuous functions from $I$ into $E$ : Also, by $C^{2}([1-\alpha, e+$ $\beta], E):=C([1-\alpha, e+\beta], E) \times C([1-\alpha, e+\beta], E)$, we denote the product Banach space with the norm:

$$
\|(u, v)\|_{C^{2}([1-\alpha, e+\beta], E)}=\|u\|_{C([1-\alpha, e+\beta], E)}+\|v\|_{C([1-\alpha, e+\beta], E)} .
$$

Let $(E, w)=\left(E, \sigma\left(E, E^{*}\right)\right)$ be the Banach space $E$ with its weak topology.

\section{Definition 2.1}

A Banach space $X$ is called Weakly Compactly Generated (WCG, in short) if it contains a weakly compact set whose linear span is dense in $X$.

\section{Definition 2.2}

A function $h: E \rightarrow E$ is said to be weakly sequentially continuous if $h$ takes each weakly convergent sequence in $E$ to a weakly convergent sequence in $E$ (i.e., for any $\left(u_{n}\right)$ in $E$ with $u_{n} \rightarrow u$ in $(E, w)$ then $h\left(u_{n}\right) \rightarrow h(u)$ in $\left.(E, w)\right)$.

\section{Definition 2.3.}

Pettis (1938) the function $u: I \rightarrow E$ is said to be Pettis integrable on $I$ if and only if there is an element $u_{J} \in E$ corresponding to each $J \subset I$ such that $\phi\left(u_{J}\right)=\int_{J} \phi(u(s)) d s$ for all $\phi \in E^{*}$, where the integral on the right hand side is assumed to exist in the sense of Lebesgue, (by definition, $\left.u_{J}=\int_{J} u(s) d s\right)$.

Let $P(I, E)$ be the space of all $E$-valued Pettis integrable functions on $I$ and denote by $L^{1}(I, E)$, the Banach space of measurable and Bochner integrable functions $u: I \rightarrow E$. Define the normed space $P_{1}(I, E)$ by:

$$
P_{1}(I, E)=\left\{u \in P(I, E): \varphi(u) \in L^{1}(I, E) ; \text { for every } \varphi \in E^{*}\right\},
$$

with the norm:

$$
\|u\|_{P_{1}}={ }_{\varphi \in E^{*},\|\varphi\| \leq 1} \int_{1}^{e}|\varphi(u(x))| d \lambda x
$$

where, $\lambda$ stands for a Lebesgue measure on $I$.

The following result is due to Pettis (1938, Theorem 3.4 and Corollary 3.41]).

\section{Proposition 2.4.}

Pettis (1938) if $u \in P_{1}(I, E)$ and $h$ is a measurable and essentially bounded $E$-valued function, then $u h \in P_{1}(J, E)$.

For all what follows, the sign " $\int "$ denotes the Pettis integral.

Let us recall some definitions and properties of Hadamard fractional integration and differentiation. We refer to (Hadamard, 1892; Kilbas et al., 2006) for a more detailed analysis.

\section{Definition 2.5.}

Hadamard (1892; Kilbas et al., 2006) The Hadamard fractional integral of order $q>0$ for a function $g \in L_{1}(I$, $E)$, is defined as:

$$
\left({ }^{H} I_{1}^{q} g\right)(x)=\frac{1}{\Gamma(q)} \int_{1}^{x}\left(\ln \frac{x}{s}\right)^{q-1} \frac{g(s)}{s} d s,
$$

where, $\Gamma(\cdot)$ is the (Euler's) Gamma function defined by:

$$
\Gamma(\xi)=\int_{0}^{\infty} t^{\xi-1} e^{-t} d t ; \xi>0
$$

Provided the integral exists.

Let $g \in P_{1}(I, E)$. For every $\varphi \in E^{*}$, we have:

$$
\varphi\left({ }^{H} I_{1}^{q} g\right)(t)=\left({ }^{H} I_{1}^{q} \varphi g\right)(t) ; \text { for a.e. } t \in I
$$

Analogously to the Riemann-Liouville fractional calculus, the Hadamard fractional derivative is defined in terms of the Hadamard fractional integral in the following way. Set:

$$
\delta=x \frac{d}{d x}, n=[q]+1,
$$

where, $[q]$ is the integer part of $q>0$ and:

$$
A C_{\delta}^{n}:=\left\{u: I \rightarrow E: \delta^{n-1}[u(x)] \in A C(I)\right\} .
$$

\section{Definition 2.6.}

Hadamard (1892; Kilbas et al., 2006) the Hadamard fractional derivative of order $q$ of a function $w \in A C_{\delta}^{n}$ is defined as: 


$$
\begin{aligned}
& \left({ }^{H} D_{1}^{q} w\right)(x)=\delta^{n}\left({ }^{H} I_{1}^{n-q} w\right)(x) \\
& =\frac{1}{\Gamma(n-q)}\left(t \frac{d}{d t}\right)^{n}{ }_{1}^{t}\left(\log \frac{t}{s}\right)^{n-q-1} \frac{h(s)}{s} d s .
\end{aligned}
$$

\section{Corollary 2.7.}

Kilbas et al. (2006) let $q>0$ and $n=[q]+1$. The equality $D^{\alpha} h(t)=0$ is valid if and only if:

$$
h(t)={ }_{j=1}^{n} c_{j}(\log t)^{q-j} \text { for each } t \in I,
$$

where, $c_{j} \in \mathbb{R}(j=1, \ldots, n)$ are arbitrary constants.

We need the following auxiliary Lemma:

\section{Lemma 2.8.}

Agarwal et al. (2016) let $1<r \leq 2, \phi \in C([1-\alpha, 1], E)$ with $\phi(1)=0, \psi \in C([e, e+\beta], E)$ with $(e)=0$ and $\sigma: I \rightarrow$ $E$ be a continuous function. The linear problem:

$$
\left\{\begin{array}{l}
D^{r} u(t)=\sigma(t) ; t \in I, \\
u(t)=\phi(t) ; t \in[1-\alpha, 1] \\
u(t)=\psi(t), t \in[e, e+\beta]
\end{array}\right.
$$

has the following unique solution:

$$
u(t)=\left\{\begin{array}{l}
\phi(t), \text { if } t \in[1-\alpha, 1], \\
-{ }_{1}^{e} G(t, s) \frac{\sigma(s)}{s} d s, \text { if } t \in I \\
\psi(t), \text { if } t \in[e, e+\beta],
\end{array}\right.
$$

where:

$$
G(t, s)=1 \Gamma(r)\left\{\begin{array}{l}
(\log t)^{r-1}(1-\log s)^{r-1} \\
-(\log t-\log s)^{r-1} ; 1 \leq s \leq t \leq e, \\
(\log t)^{r-1}(1-\log s)^{r-1} ; 1 \leq t \leq s \leq e .
\end{array}\right.
$$

\section{Definition 2.9.}

De Blasi (1977) let $E$ be a Banach space, $\Omega_{E}$ the bounded subsets of $E$ and $B_{1}$ the unit ball of $E$. The De Blasi measure of weak noncompactness is the map $\mu$ : $\Omega_{E} \rightarrow[0, \infty)$ defined by $\mu(X)=\inf \{\varepsilon>0$ : There exists a weakly compact subset $\Omega$ of $E: X \subset \varepsilon B_{1}+\Omega$ \}.

The De Blasi measure of weak noncompactness satisfies the following properties:

- $A \subset B \Rightarrow \mu(A) \leq \mu(B)$

- $\mu(A)=0 \Leftrightarrow A$ is weakly relatively compact

- $\mu(A \cup B)=\max \{\mu(A), \mu(B)\}$

- $\mu\left(\bar{A}^{\omega}\right)=\mu(A),\left(\bar{A}^{\omega}\right.$ denotes the weak closure of $\left.A\right)$

- $\mu(A+B) \leq \mu(A)+\mu(B)$

- $\mu(\lambda A)=|\lambda| \mu(A)$
- $\mu(\operatorname{conv}(A))=\mu(A)$

- $\mu\left(\bigcup_{|\lambda| \leq h} \lambda A\right)=h \mu(A)$

The next result follows directly from the HahnBanach theorem.

\section{Proposition 2.10}

Let $E$ be a normed space and $x_{0} \in E$ with $x_{0} \neq 0$. Then, there exists $\varphi \in E^{*}$ with $\|\varphi\|=1$ and $\varphi\left(x_{0}\right)=\left\|x_{0}\right\|$.

For a given set $V$ of functions $v: I \rightarrow E$ let us denote by:

$$
V(t)=\{v(t): v \in V\} ; t \in I,
$$

and:

$$
V(I)=\{v(t): v \in V, t \in I\}
$$

\section{Lemma 2.11.}

Guo et al. (1996) let $H \subset C$ be a bounded and equicontinuous. Then the function $t \rightarrow \mu(H(t))$ is continuous on $I$ and:

$$
\mu_{C}(H)=_{t \in I} \mu(H(t)),
$$

and:

$$
\mu\left(\int_{I} u(s) d s\right) \leq \int_{I} \mu(H(s)) d s
$$

where, $H(t)=\{u(t): u \in H\}, t \in I$ and $\mu_{C}$ is the De Blasi measure of weak noncompactness defined on the bounded sets of $C$.

For our purpose we will need the following fixed point theorem:

\section{Theorem 2.12.}

O'Regan (1998) let $Q$ be a nonempty, closed, convex and equicontinuous subset of a metrizable locally convex vector space $C(I)$ such that $0 \in Q$. Suppose $T: Q \rightarrow Q$ is weakly-sequentially continuous. If the implication:

$\bar{V}=\operatorname{conv}(\{0\} \cup T(V)) \Rightarrow V$ is relatively weakly compact,

holds for every subset $V \subset Q$, then the operator $T$ has a fixed point.

\section{Existence of Weak Solutions}

Definition 3.1.

A coupled functions $(u, v) \in C^{2}([1-\alpha, e+\beta], E)$ is said to be a weak solution of the system $(2)$ if $(u, v)$ satisfies the equations $\left({ }^{H} D_{1}^{r_{1}} u\right)(t)=f_{1}\left(t, u_{t}, v_{t}\right)$ and $\left({ }^{H} D_{1}^{r_{2}} v\right)(t)=$ 
$f_{2}\left(t, u_{t}, v_{t}\right)$ on $I$ and the conditions $(u(t), v(t))=\left(\phi_{1}(t)\right.$, $\left.\phi_{2}(t)\right) ; \phi_{i}(1)=0 ; i=1,2$ on $[1-\alpha, 1]$ and $(u(t), v(t))=$ $\left(\psi_{1}(t), \psi_{2}(t)\right) ; \psi_{i}(e)=0 ; i=1,2$ on $[e, e+\beta]$.

Let us introduce the following hypotheses:

$\left(H_{1}\right)$ For a.e. $t \in I$, the functions $v \rightarrow f_{i}(t, u, \cdot)$ and $v \rightarrow$ $f_{i}(t, \cdot, v) ; i=1,2$ are weakly sequentially continuous

$\left(H_{2}\right)$ For a.e. $u, v \in C[1-\alpha, e+\beta]$, the functions $t \rightarrow f_{i}(t$, $u, v)$ are Pettis integrable a.e. on $I$

$\left(H_{3}\right)$ There exist $p_{i} \in C(I,[0, \infty))$ such that for all $\varphi \in E^{*}$ :

$$
\left|\varphi\left(f_{i}(t, u, v)\right)\right| \leq \frac{p_{i}(t)\|\varphi\|}{1+\|\varphi\|+\left\|u_{t}\right\|_{C[-\alpha, \beta]}+\left\|v_{t}\right\|_{C[-\alpha, \beta]}}
$$

for a.e. $t \in I$ and each $u, v \in C[1-\alpha, e+\beta]$,

$\left(H_{4}\right)$ For each bounded and measurable set $B \subset C^{2}[1-\alpha, e$ $+\beta$ ] and for each $t \in I$, we have:

$$
\begin{aligned}
& \mu\left(f_{1}(t, B), 0\right) \leq p_{1}(t) \mu(B), \text { and } \\
& \mu\left(0, f_{2}(t, B)\right) \leq p_{2}(t) \mu(B),
\end{aligned}
$$

where:

$$
\left(f_{1}(t, B), 0\right)=\left(f_{1}\left(t, v_{1}(t), v_{2}(t)\right), 0\right) ;\left(v_{1}, v_{2}\right) \in B,
$$

and:

$$
\left(0, f_{2}(t, B)\right)=\left\{\left(0, f_{2}\left(t, v_{1}(t), v_{2}(t)\right)\right) ;\left(v_{1}, v_{2}\right) \in B\right\} .
$$

Set:

$$
p_{i}^{*}=_{t \in I} p_{i}(t) ; i=1,2 .
$$

Now we are able to state and prove our main result throughout the following theorem.

\section{Theorem 3.2.} hold:

Assume that $\left(H_{1}\right)-\left(H_{4}\right)$ and the following condition

$$
L:=\frac{2 p_{1}^{*}}{\Gamma\left(1+r_{1}\right)}+\frac{2 p_{2}^{*}}{\Gamma\left(1+r_{2}\right)}<1,
$$

Then the coupled system (2) has at least one weak solution defined on $[1-\alpha, e+\beta]$.

\section{Proof}

Define the operators $N_{1}, N_{2}: C[1-\alpha, e+\beta] \rightarrow C[1-\alpha$, $e+\beta$ ] by:

$$
\left(N_{1} u\right)(t)=\left\{\begin{array}{l}
\phi_{1}(t) ; t \in[1-\alpha, 1], \\
-{ }_{1}^{e} G_{1}(t, s) \frac{f_{1}\left(s, u_{s}, v_{s}\right)}{s} d s ; t \in I, \\
\psi_{1}(t) ; t \in[e, e+\beta],
\end{array}\right.
$$

and:

$$
\left(N_{1} v\right)(t)=\left\{\begin{array}{l}
\phi_{2}(t) ; t \in[1-\alpha, 1], \\
-{ }_{1}^{e} G_{2}(t, s) \frac{f_{2}\left(s, u_{s}, v_{s}\right)}{s} d s ; t \in I, \\
\psi_{2}(t) ; t \in[e, e+\beta],
\end{array}\right.
$$

where:

$$
\begin{aligned}
& G_{i}(t, s) \\
& =\frac{1}{\Gamma\left(r_{i}\right)}\left\{\begin{array}{l}
(\log t)^{r_{i}-1}(1-\log s)^{r_{i}-1} \\
-(\log t-\log s)^{r_{i}-1} ; 1 \leq s \leq t \leq e, \quad ; i=1,2 . \\
(\log t)^{r_{i}-1}(1-\log s)^{r_{i}-1} ; 1 \leq t \leq s \leq e,
\end{array}\right.
\end{aligned}
$$

Consider the continuous operator $N: C^{2}[1-\alpha, e+\beta]$ $\rightarrow C^{2}[1-\alpha, e+\beta]$ defined by:

$$
\begin{aligned}
& (N(u, v))(t)=\left(\left(N_{1} u\right)(t),\left(N_{2} v\right)(t)\right) \\
& =\left\{\begin{array}{l}
\left(\phi_{1}(t), \phi_{2}(t)\right) ; t \in[1-\alpha, 1], \\
\left(-{ }_{1}^{e} G_{1}(t, s) \frac{f_{1}\left(s, u_{s}, v_{s}\right)}{s} d s,-{ }_{1}^{e} G_{2}(t, s) \frac{f_{2}\left(s, u_{s}, v_{s}\right)}{s} d s\right) ; t \in I, \\
\psi_{1}(t), \psi_{2}(t) ; t \in[e, e+\beta] .
\end{array}\right.
\end{aligned}
$$

First notice that, the functions $\phi_{i}$ and $\psi_{i} ; i=1,2$ are continuous and the hypotheses imply that for all $t \in I$, the functions $t \mapsto G(\cdot, t)$ and $t \mapsto f_{1}\left(s, u_{s}, v_{s}\right)$ are Pettis integrable, over $I$. Thus, the operator $N$ is well defined.

In the following we denote $\|w\|_{C[1-\alpha, e+\beta]}$ by $\|w\|_{C}$.

Let $R>0$ be such that $R=R_{1}+R_{2}$, with:

$$
R_{i}>\left\{\frac{2 p_{i}^{*}}{\Gamma\left(1+r_{i}\right)},\left\|\phi_{i}\right\|_{C[1-\alpha, 1]},\left\|\psi_{i}\right\|_{C[e, e+\beta]}\right\} ; i=1,2,
$$

and consider the closed, convex and equicontinuous subset $Q$ of $C_{2}[1-\alpha, e+\beta]$ defined by:

$$
Q=\left\{\begin{array}{l}
(u, v) \in C^{2}[1-\alpha, e+\beta]:\|(u, v)\|_{C^{2}[1-\alpha, e+\beta]} \\
\leq R,\left\|u\left(t_{2}\right)-u\left(t_{1}\right)\right\|_{E} \\
\leq p_{11}^{* e}\left|G_{1}\left(t_{2}, s\right)-G_{1}\left(t_{1}, s\right)\right| \frac{d s}{s}, \text { and } \| v\left(t_{2}\right)-v\left(t_{1}\right) \\
\|_{E} \leq p_{21}^{* e}\left|G_{2}\left(t_{2}, s\right)-G_{2}\left(t_{1}, s\right)\right| \frac{d s}{s}
\end{array}\right\} .
$$

We shall show that the operator $N$ satisfies all the assumptions of Theorem 2.12. The proof will be given in several steps.

Step 1. $N$ maps $Q$ into itself.

Let $(u, v) \in Q ; t \in I$ and assume that $(N(u, v))(t) \neq(0,0)$. Then there exists $\varphi_{i} \in E^{*} ; i=1,2$ such that for each $t \in I$, we have: 


$$
\|(N(u, v))(t)\|_{E}=\left(\varphi_{1}\left(\mid\left(N_{1} u\right)(t)\right), \varphi_{2}\left(\mid\left(N_{2} v\right)(t)\right)\right) .
$$

Thus:

$$
\left\|\left(N_{1} u\right)(t)\right\|_{E}=\varphi_{1}\left({ }_{1}^{e} G_{1}(t, s) f_{1}\left(s, u_{s}, v_{s}\right) \frac{d s}{s}\right) .
$$

If $t \in[1-\alpha, 1]$, then:

$$
\left\|N_{1}(u)(t)\right\|_{E} \leq\left\|\phi_{1}\right\|_{[1-\alpha, 1]} \leq R_{1},
$$

also, if $t \in[e, e+\beta]$, then:

$$
\left\|N_{1}(u)(t)\right\|_{E} \leq\left\|\psi_{1}\right\|_{[e, e+\beta]} \leq R_{1} .
$$

For each $t \in I$ and any $i=1,2$, we have:

$$
\begin{aligned}
& { }_{1}^{e}\left|G_{i}(t, s)\right| \frac{d s}{s} \leq \frac{1}{\Gamma\left(r_{i}\right)}\left[\begin{array}{l}
t \\
{[}
\end{array}\left(\log \frac{t}{s}\right)^{r_{i}-1} \frac{d s}{s}+(\log t)_{1}^{r_{i}-1} e\left(\log \frac{e}{s}\right)^{r_{i}-1} \frac{d s}{s}\right] \\
& \leq \frac{2}{\Gamma\left(r_{i}\right)_{1}}\left(\log \frac{e}{s}\right)^{r_{i}-1} d s s=\frac{2}{\Gamma\left(1+r_{i}\right)} .
\end{aligned}
$$

Thus, for each $t \in I$, we have:

$$
\begin{aligned}
& \left\|\left(N_{1} u\right)(t)\right\|_{E} \leq \int_{1}^{e}\left|G_{1}(t, s)\right| \frac{\left|\varphi\left(f_{1}\left(s, u_{s}, v_{s}\right)\right)\right|}{s} d s \\
& \leq \frac{2 p_{1}^{*}}{\Gamma\left(1+r_{1}\right)} \\
& \leq R_{1},
\end{aligned}
$$

Hence, for each $t \in[1-\alpha, e+\beta]$, we have:

$$
\left\|\left(N_{1} u\right)(t)\right\|_{E} \leq R_{1} .
$$

Also, for each $t \in[1-\alpha, e+\beta]$, we obtain:

$$
\left\|\left(N_{2} v\right)(t)\right\|_{E} \leq R_{2} .
$$

Therefore, for each $t \in[1-\alpha, e+\beta]$ and all $(u, v) \in Q$, we get:

$$
\|\left(N(u, v)(t) \|_{E} \leq R_{1}+R_{2}=R .\right.
$$

Next, let $t_{1}, t_{2} \in I$ such that $t_{1}<t_{2}$ and let $(u, v) \in Q$, with:

$$
(N(u, v))\left(t_{2}\right)-(N(u, v))\left(t_{1}\right) \neq(0,0)
$$

Then there exists $\varphi_{i} \in E^{*}, i=1,2$ with $\left\|\varphi_{1}\right\|=1$ such that:

$$
\left\|\left(N_{1} u\right)\left(t_{2}\right)-\left(N_{1} u\right)\left(t_{1}\right)\right\|_{E}=\varphi_{1}\left(\left(N_{1} u\right)\left(t_{2}\right)-\left(N_{1} u\right)\left(t_{1}\right)\right),
$$

and:

$$
\left\|\left(N_{2} v\right)\left(t_{2}\right)-\left(N_{2} v\right)\left(t_{1}\right)\right\|_{E}=\varphi_{2}\left(\left(N_{2} v\right)\left(t_{2}\right)-\left(N_{2} v\right)\left(t_{1}\right)\right) .
$$

Thus:

$$
\begin{aligned}
& \left\|\left(N_{1} u\right)\left(t_{2}\right)-\left(N_{1} u\right)\left(t_{1}\right)\right\|_{E}=\varphi_{1}\left(\left(N_{1} u\right)\left(t_{2}\right)-\left(N_{1} u\right)\left(t_{1}\right)\right) \\
& \leq \varphi_{1}\left(e\left(G_{1}\left(t_{2}, s\right)-G_{1}\left(t_{1}, s\right)\right) f_{1}\left(s, u_{s}, v_{s}\right) \frac{d s}{s}\right)
\end{aligned}
$$

This gives:

$$
\begin{aligned}
& \left\|\left(N_{1} u\right)\left(t_{2}\right)-\left(N_{1} u\right)\left(t_{1}\right)\right\|_{E}=e_{1}^{e} \mid G_{1}\left(t_{2}, s\right) \\
& -G_{1}\left(t_{1}, s\right) \| f_{1}\left(s, u_{s}, v_{s}\right) \mid \frac{d s}{s} \\
& \leq p_{11}^{* e}\left|G_{1}\left(t_{2}, s\right)-G_{1}\left(t_{1}, s\right)\right| \frac{d s}{s} .
\end{aligned}
$$

Also, we can obtain:

$$
\begin{aligned}
& \left\|\left(N_{2} v\right)\left(t_{2}\right)-\left(N_{2} v\right)\left(t_{1}\right)\right\|_{E}==_{1}^{e} \mid G_{2}\left(t_{2}, s\right) \\
& -G_{2}\left(t_{1}, s\right) \| f_{2}\left(s, u_{s}, v_{s}\right) \mid \frac{d s}{s} \\
& \leq p_{21}^{*}\left|G_{2}\left(t_{2}, s\right)-G_{2}\left(t_{1}, s\right)\right| \frac{d s}{s} .
\end{aligned}
$$

Hence $N(Q) \subset Q$.

Step 2. $N$ is weakly-sequentially continuous.

Let $\left(u_{n}, v_{n}\right)$ be a sequence in $Q$ and let $\left(u_{n}(t)\right.$, $\left.v_{n}(t)\right) \rightarrow(u(t), v(t))$ in $(E, \omega) \times(E, \omega)$ for each $t \in[1-\alpha, e+$ $\beta]$. Fix $t \in[1-\alpha, e+\beta]$, since the functions $f_{i} ; i=1,2$ satisfy the assumption $\left(H_{1}\right)$, we have $f_{i}\left(t, u_{n t}, v_{n t}\right)$ converge weakly uniformly to $f_{i}\left(t, u_{t}, v_{t}\right)$. Hence the Lebesgue dominated convergence theorem for Pettis integral implies $\left(N\left(u_{n}, v_{n}\right)\right)(t)$ converges weakly uniformly to $(N(u, v))(t)$ in $(E, \omega) \times(E, \omega)$, for each $t \in[1-\alpha, e+\beta]$. Thus, $N\left(u_{n}, v_{n}\right) \rightarrow N(u, v)$. Hence, $N: Q$ $\rightarrow Q$ is weakly-sequentially continuous.

Step 3. The implication (4) holds.

Let $V$ be a subset of $Q$ such that $\bar{V}=\overline{\operatorname{conv}}(N(V) \cup\{(0$, $0)\}$ ). Obviously:

$$
V(t) \subset \overline{\operatorname{conv}}(N V(t)) \cup\{(0,0)\}, \text { for all } t \in[1-\alpha, e+\beta] .
$$

Further, as $V$ is bounded and equicontinuous, by Lemma 3 in (Bugajewski and Szua, 1993), the function $t$ $\rightarrow \mu(V(t))$ is continuous on $[1-\alpha, e+\beta]$.

It is clear that for any $t \in[1-\alpha, 1] \cup[e, e+\beta]$, the set $V$ is weakly relatively compact. Next, from $\left(H_{3}\right),\left(H_{4}\right)$, Lemma 2.11 and the properties of the measure $\mu$, for any $t \in I$, we have: 


$$
\begin{aligned}
\mu(V(t)) \leq & \mu((N V)(t) \cup\{(0,0)\}) \\
& \leq \mu((N V)(t)) \\
& \left.=\mu\left(\left\{\left(N_{1} v_{1}\right)(t),\left(N_{2} v_{2}\right)(t)\right) ;\left(v_{1}, v_{2}\right) \in V\right\}\right) \\
& \leq \int_{1}^{c} \mu\left(\left\{\left(G_{1}(t, s) f_{1}\left(s, v_{1}(s), v_{2}(s)\right), G_{2}(t, s) f_{2}\left(s, v_{1}(s), v_{2}(s)\right)\right) ;\left(v_{1}, v_{2}\right) \in V\right\}\right) \frac{d s}{s} \\
& \leq \int_{1}^{e}\left|G_{1}(t, s)\right| \mu\left(\left\{\left(f_{1}\left(s, v_{1}(s), v_{2}(s)\right), 0\right) ;\left(v_{1}, v_{2}\right) \in V\right\}\right) \frac{d s}{s} \\
& +\int_{1}^{e}\left|G_{2}(t, s)\right| \mu\left(\left\{\left(0, f_{2}\left(s, v_{1}(s), v_{2}(s)\right)\right) ;\left(v_{1}, v_{2}\right) \in V\right\}\right) \frac{d s}{s} \\
& \leq \int_{1}^{e}\left|G_{1}(t, s)\right| p_{1}(s) \mu\left(\left\{\left(v_{1}(s), v_{2}(s)\right) ;\left(v_{1}, v_{2}\right) \in V\right\}\right) \frac{d s}{s} \\
& +\int_{1}^{e}\left|G_{2}(t, s)\right| p_{2}(s) \mu\left(\left\{\left(v_{1}(s), v_{2}(s)\right) ;\left(v_{1}, v_{2}\right) \in V\right\}\right) \frac{d s}{s} \\
\leq & \int_{1}^{e}\left|G_{1}(t, s)\right| p_{1}(s) \mu(V(s)) \frac{d s}{s}+\int_{1}^{e}\left|G_{2}(t, s)\right| p_{2}(s) \mu(V(s)) \frac{d s}{s} \\
\leq & \left(p_{1}^{*} \int_{1}^{e}\left|G_{1}(t, s)\right| \frac{d s}{s}+p_{2}^{*} \int_{1}^{e}\left|G_{2}(t, s)\right| \frac{d s}{s}\right)_{t \in I} \mu(V(t)) \\
\leq & \left(\frac{2 p_{1}^{*}}{\Gamma\left(1+r_{1}\right)}+\frac{2 p_{2}^{*}}{\Gamma\left(1+r_{2}\right)}\right)_{t \in I} \mu(V(t)) \\
= & L_{t \in I} \mu(V(t)) .
\end{aligned}
$$

Thus:

$$
{ }_{t \in I} \mu(V(t)) \leq L_{t \in I} \mu(V(t)) .
$$

Hence, the inequality (5) implies that ${ }_{t \in I} \mu(V(t))=0$. This shows $\mu(V(t))=0$; for each $t \in I$. Then from Theorem 2 in (Mitchell and Smith, 1977), we conclude that the set $V$ is weakly relatively compact in $C^{2}[1-\alpha, e+$ $\beta]$. Therefore, Theorem 2.12 implies that $N$ has a fixed point which is a solution of the coupled system (2).

\section{An Example}

Let:

$$
E=l^{1}=\left\{u=\left(u_{1}, u_{2}, \ldots, u_{n}, \ldots\right)_{{ }_{n=1}}^{\infty}\left|u_{n}\right|<\infty\right\}
$$

be the Banach space with the norm:

$$
\|u\|_{E}={ }_{n=1}^{\infty}\left|u_{n}\right| .
$$

Consider the following coupled system of Hadamard fractional differential equations of the form:

$$
\left\{\begin{array}{l}
(u(t), v(t))=\left(1-e^{t-1}, 0\right) ; t \in[-2,1] \\
\left(\left({ }^{H} D_{1}^{\frac{3}{2}} u_{n}\right)(t),\left({ }^{H} D_{1}^{\frac{3}{3}} v_{n}\right)(t)\right)=\left(f_{n}\left(t, u_{t}, v_{t}\right), g_{n}\left(t, u_{t}, v_{t}\right)\right) ; t \in[1, e],(10) \\
(u(t), v(t))=(-1+\ln t, 0) ; t \in[e, 2 e],
\end{array}\right.
$$$$
f_{n}\left(t, u_{t}, v_{t}\right)
$$$$
=\frac{c t^{2}}{1+\|u\|_{C[-3, e]}+\|v\|_{C[-3, e]}}\left(e^{-7}+\frac{1}{e^{t+5}}\right) u_{n}(t) ; t \in[1, e] \text {, }
$$

and:

$$
g_{n}\left(t, u_{t}, v_{t}\right)=\frac{c t^{2} e^{-6}}{1+\|u\|_{C[-3, e]}+\|v\|_{C[-3, e]}} ; t \in[1, e],
$$

with:

$$
u=\left(u_{1}, u_{2}, \ldots, u_{n}, \ldots\right), v=\left(v_{1}, v_{2}, \ldots, v_{n}, \ldots\right), \text { and } c:=\frac{e^{4}}{24} \Gamma\left(\frac{1}{2}\right) .
$$

Set:

$$
f=\left(f_{1}, f_{2}, \ldots, f_{n}, \ldots\right), g=\left(g_{1}, g_{2}, \ldots, g_{n}, \ldots\right) .
$$

Clearly, the functions $f$ and $g$ are continuous.

For each $u, v \in E$ and $t \in[1, e]$, we have:

$$
\left\|f\left(t, u_{t}, v_{t}\right)\right\|_{E} \leq \frac{c t^{2}}{1+\|u\|_{C[-3, e]}+\|v\|_{C[-3, e]}}\left(e^{-7}+\frac{1}{e^{t+5}}\right),
$$

and:

$$
\left\|g\left(t, u_{t}, v_{t}\right)\right\|_{E} \leq \frac{c t^{2} e^{-6}}{1+\|u\|_{C[-3, e]}+\|v\|_{[[-3, e]}} .
$$

where: 
Hence, the hypothesis $\left(H_{3}\right)$ is satisfied with $p_{1}^{*}=p_{2}^{*}=c e^{-4}$.

We shall show that condition (5) holds. Indeed:

$$
\frac{2 p_{1}^{*}}{\Gamma\left(1+r_{1}\right)}+\frac{2 p_{2}^{*}}{\Gamma\left(1+r_{2}\right)}=\frac{c}{e^{4} \Gamma\left(\frac{5}{2}\right)}=\frac{1}{2}<1 .
$$

Simple computations show that all conditions of Theorem 3.2 are satisfied. It follows that the coupled system (10) has at least one solution on $[-2,2 e]$.

\section{Acknowledgement}

The authors are grateful to the editor and the referees for their helpful remarks.

\section{Author's Contributions}

The paper was realized in complete collaboration. All authors have read and approved the final manuscript.

\section{Ethics}

The Authors declare there is not conflict of interest.

\section{References}

Abbas, S., M. Benchohra, J. Lazreg and G. N'Guerekata, 2017. Weak solutions for implicit Pettis Hadamard fractional differential equations with retarded and advanced arguments. Nonlinear Stud., 24: 355-365.

Abbas, S., M. Benchohra and G.M. N'Guerekata, 2012. Topics in Fractional Differential Equations. 1st Edn., Springer, New York.

Abbas, S., M. Benchohra and G.M. N'Guerekata, 2015. Advanced Fractional Differential and Integral Equations. 1st Edn., Nova Science Publishers, New York.

Agarwal, R.P., S.K. Ntouyas, B. Ahmad and A.K. Alzahrani, 2016. Hadamard-type fractional functional differential equations and inclusions with retarded and advanced arguments. Adv. Diff. Equ., 2016: 92-92.

Ahmad, B., A. Alsaedi, S.K. Ntouyas and J. Tariboon, 2017. Hadamard-type fractional differential equations, inclusions and inequalities. Springer, Cham.

Ahmad, B. and S.K. Ntouyas, 2015. Initial value problem of fractional order Hadamard-type functional differential equations. Electron. J. Diff. Equ., 2015: 1-9.

Akhmerov, R.R., M.I. Kamenskii, A.S. Patapov, A.E. Rodkina and B.N. Sadovskii, 1992. Measures of Noncompactness and Condensing Operators. 1st Edn., Birkhauser Verlag, Basel.
Alvarez, J.C., 1985. Measure of noncompactness and fixed points of nonexpansive condensing mappings in locally convex spaces. Rev. Real. Acad. Cienc. Exact. Fis. Natur. Madrid, 79: 53-66.

Banas, J. and K. Goebel, 1980. Measures of Noncompactness in Banach Spaces. 1st Edn., Marcel Dekker, New York.

Benchohra, M., J. Graef and F.Z. Mostefai, 2011. Weak solutions for boundary-value problems with nonlinear fractional differential inclusions. Nonlinear Dyn. Syst. Theory, 11: 227-237.

Benchohra, M., J. Henderson and F.Z. Mostefai, 2012. Weak solutions for hyperbolic partial fractional differential inclusions in Banach spaces. Comput. Math. Applied, 64: 3101-3107.

Benchohra, M., J. Henderson and D. Seba, 2008. Measure of noncompactness and fractional differential equations in Banach spaces. Commun. Applied Anal., 12: 419-428.

Bugajewski, D. and S. Szua, 1993. Kneser's theorem for weak solutions of the Darboux problem in a Banach space. Nonlinear Anal., 20: 169-173.

De Blasi, F.S., 1977. On a property of the unit sphere in a Banach space. Bull. Math. Soc. Sci. Math. R.S. Roumanie, 21: 259-262.

Guo, D., V. Lakshmikantham and X. Liu, 1996. Nonlinear Integral Equations in Abstract Spaces. 1st Edn., Kluwer Academic Publishers, Dordrecht.

Hadamard, J., 1892. Essai sur l'etude des functions donnees par leur development de Taylor. J. Pure Applied Math., 4: 101-186.

Hilfer, R., 2000. Applications of Fractional Calculus in Physics. 1st Edn., World Scientific, Singapore.

Kilbas, A.A., 2001. Hadamard-type fractional calculus. J. Korean Math. Soc., 38: 1191-1204.

Kilbas, A.A., H.M. Srivastava and J.J. Trujillo, 2006. Theory and Applications of Fractional Differential Equations. 1st Edn., Elsevier Science B.V., Amsterdam.

Mitchell, A.R. and C. Smith, 1977. An existence theorem for weak solutions of differential equations in Banach spaces. Nonlinear equations in abstract spaces. Proceedings of the International Symposium, Academic Press, New York, pp: 387-403.

O'Regan, D., 1998. Fixed point theory for weakly sequentially continuous mapping. Math. Comput. Model., 27: 1-14.

O'Regan, D., 1999. Weak solutions of ordinary differential equations in Banach spaces. Applied Math. Lett., 12: 101-105.

Pettis, B.J., 1938. On integration in vector spaces. Trans. Am. Math. Soc., 44: 277-304.

Reich, S., 1973. Fixed points of condensing functions. J. Math. Anal. Applied, 41: 460-467. 
Reich, S., 1972. Fixed points in locally convex spaces. Math. Z., 125: 17-131.

Samko, S.G., A.A. Kilbas and O.I. Marichev, 1993. Fractional Integrals and Derivatives: Theory and Applications. 1st Edn., Gordon and Breach, Yverdon.

Tarasov, V.E., 2010. Fractional dynamics: Application of Fractional Calculus to Dynamics of Particles, Fields and Media. 1st Edn., Higher Education Press, Beijing.
Thiramanus, P., S.K. Ntouyas and J. Tariboon, 2014. Existence and uniqueness results for Hadamard-type fractional differential equations with nonlocal fractional integral boundary nonditions, Abst. Applied Anal.

Zhou, Y., 2014. Basic Theory of Fractional Differential Equations. 1st Edn., World Scientific, Singapore. 